\title{
Low Complexity Algorithm for Robust Video Frame Rate Up-Conversion (FRUC) Technique
}

\author{
Tanaphol Thaipanich, Student Member, IEEE, Ping-Hao Wu, Student Member, IEEE, \\ and C.-C. Jay Kuo Fellow, IEEE
}

\begin{abstract}
Two challenging situations for video frame rate up-conversion (FRUC) are first identified and analyzed; namely, when the input video has abrupt illumination change and/or a low frame rate. Then, a low-complexity processing technique and robust FRUC algorithm are proposed to address these two issues. The proposed algorithm utilizes a translational motion vector model of the first-and the secondorder and detects the continuity of these motion vectors. Additionally, in order to improve perceptual quality of interpolated frame, spatial smoothness criterion is employed. The superior performance of the proposed algorithm has been tested extensively and representative examples are given in this work. ${ }^{l}$.
\end{abstract}

Index Terms - Frame rate up-conversion, FRUC, Abrupt illumination change, Low frame rate.

\section{INTRODUCTION}

Video frame rate-up conversion (FRUC) has been a technique of great interest due to its diversified applications in consumer electronics. For instance, HDTV and multimedia PC system usually have capability to display a much higher frame rate than that offered by broadcasting video stream [3]. In this case, FRUC can be employed to increase the original frame rate in order to enhance the viewing experience of end-user. For handheld device, when temporal mode of scalable video coding [21] is used, frame rate of transmitted video may fluctuate due to a varying available bandwidth of transmission channel. FRUC can be exploited to stabilize video frame rate under this condition. Many FRUC algorithms adopt a motion interpolation technique to determine the motion vector (MV) field of interpolated frames. In general, FRUC algorithm relies on temporal and/or spatial correlation of the original video sequence in order to construct the up-sampled frame [2] [11]. The concept of FRUC is very similar to that of video error concealment (EC). In particular, frame loss concealment aims to reconstruct the missing frame when video sequence encounters a transmission lost. Therefore, there are many fundamental ideas that have been shared by both FRUC and EC such as motion trajectory prediction, optical flow

\footnotetext{
${ }^{1}$ Part of this work was presented at ICCE2009, Las Vegas, NV, USA [1]

Tanaphol Thaipanich, Ping-hao Wu and C.-C. Jay Kuo are with the Ming Hsieh Department of Electrical Engineering and the Signal and Image Processing Institute, University of Southern California, Los Angeles, CA 90089 USA (E-mails: thaipani@usc.edu, pinghaow@usc.edu, and cckuo@sipi.usc.edu)
}

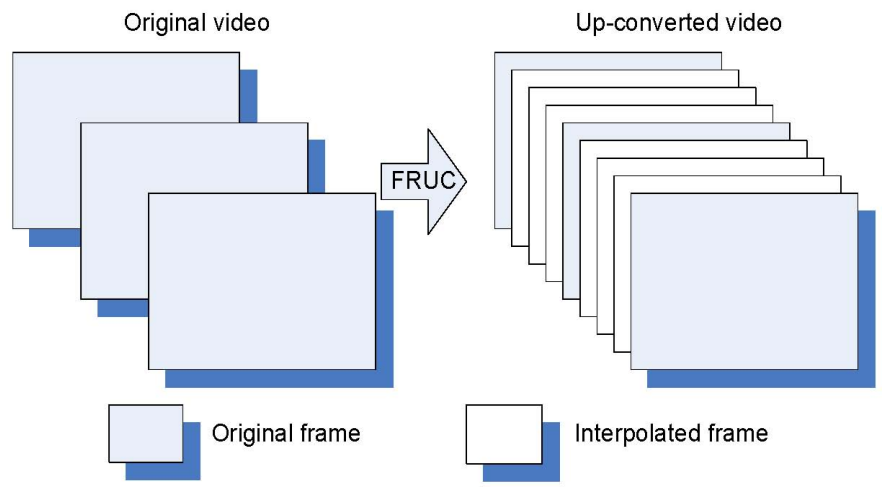

Figure 1: Frame rate up-conversion (FRUC) scheme

assumption, etc [16] - [20]. Generally speaking, today's advanced FRUC techniques provide satisfactory results for most video sequences in preserving temporal continuity. However, we observe two challenging situations, where the performance of FRUC deteriorates severely. We will address these two situations and propose a low complexity solution in this work.

Most existing FRUC algorithms fail to provide good results when the input video sequence has an abrupt illumination change (e.g., under a flashlight) and a lower frame rate (e.g., $10 \mathrm{fps}$ or lower). Under these two conditions, the estimated MV field between frames is not accurate, which results in an adverse effect on the motion interpolation process of FRUC. To address the difficulty of a low input frame rate, we propose a new FRUC algorithm that employs the first- and the secondorder translational MV model and detects the continuity of the MV field. Instead of relying solely on temporal correlation among frame, spatial smoothness criterion is also used to ensure a better perceptual quality of the up-converted video sequence. To handle abrupt illumination change, we present a low complexity technique to detect the sudden shift in lighting condition. This technique relies on a detection results obtained from applying adaptive thresholding to the first- and the second-order difference of the frame luminance histogram. Then, local intensity adjustment method is used to regulate frame intensity and consequently, allows motion estimation process to obtain a more precise object motion. The superior performance of the proposed algorithm has been tested extensively and representative examples are given in this work.

The rest of this paper is organized as follows. The effect of abrupt illumination change and low frame rate on FRUC is addressed in Sec. II. The proposed solutions for these two 
conditions are presented in Sec. III. In Sec. IV, we describe a brief detail of six other low complexity FRUC algorithms used as a benchmark for performance comparison. Associated experimental results and performance evaluations are presented in Sec. V. Finally, concluding remarks are given in Sec. VI.

\section{Problem Statement}

\section{A. Abrupt Illumination Change}

Most FRUC algorithms rely on temporal continuity of MV field in order to interpolate the up-sampled frames. Motion information obtained from motion estimation process in the encoder is not always reliable since the process aims at minimizing encoding residue instead of acquiring the actual object motion. Under an abrupt illumination change condition, motion estimation is unable to determine the correct object motion due to an irregular increase in pixel intensity and consequently, the temporal continuity of $\mathrm{MV}$ field is interrupted. This phenomenon occurs due to significant brightness variation of the environment or the camera effect such as flash, fade in/out, etc. Without proper pre-processing, motion interpolation based FRUC algorithms cannot determine an accurate MV for frame interpolation. Fig. 2(a) illustrates the MV field overlaid on the compensated frame of the crew sequence using [23]. In this instance, the temporal continuity of MV field is preserved until frame no. 30, where the camera flash occurs. The effect causes a disruption in MV field on both the current frame and the next frame that uses it as a reference. After that, the temporal continuity of MV field is gradually restored.

\section{B. Low frame rates}

When the input frame rate is low, the temporal distance of two adjacent frames become larger and, consequently, the frame correlation is drastically decreased. This behavior can be observed by examining the motion residual of the encoded video under two frame rates as shown in Fig. 3. Under a low frame rate condition, it is more difficult for motion search to get accurate MV estimation for each macroblock due to the lost information between frames. The linear translational motion assumption, which is exploited by many FRUC algorithms to interpolate up-sampled frames, may fail as the frame rate decreases. This is especially true for video with fast moving objects and inconsistent camera motion. An example is shown in Fig. 2(b). In this case, the motion information of the first three frames is consistent with the actual object motion. However, after frame no. 4, the motion vector field becomes erratic due to a fast camera panning that starts at frame no. 5. We observe that the effect of the low frame rate on FRUC starts to manifest when the frame rate is dropped below 10 fps for video with fast camera movement and object motion such as soccer, ice and football sequence. The attempt to offset this effect by increasing the search range in motion estimation is not effective since the inaccurate motion information is usually caused by a low temporal correlation among frames. The problem of FRUC under a low frame rate circumstance is a challenging study since most FRUC algorithms exploit a correlation between two adjacent frames in order to construct an interpolated frame. With a wider temporal gap, this elation reduces drastically and the motion information obtained from the motion estimation process becomes unreliable. In the next section, we propose a FRUC algorithm to handle this specific condition.

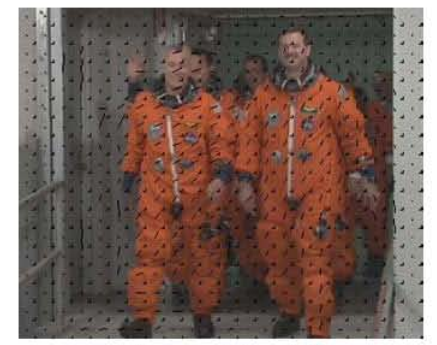

Frame No. 29

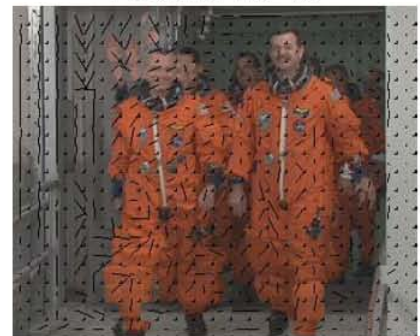

Frame No. 30

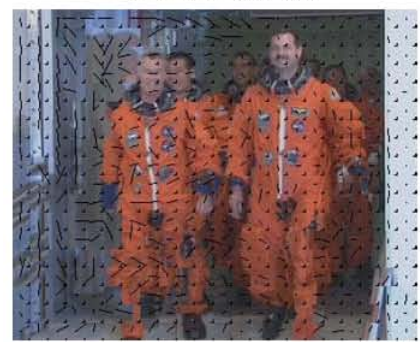

Frame No. 31

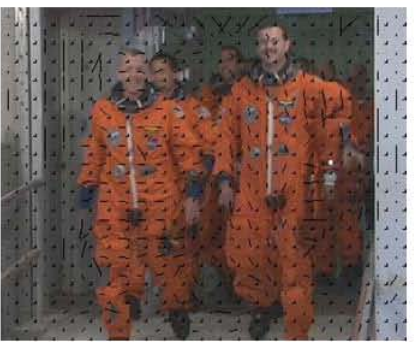

Frame No. 32

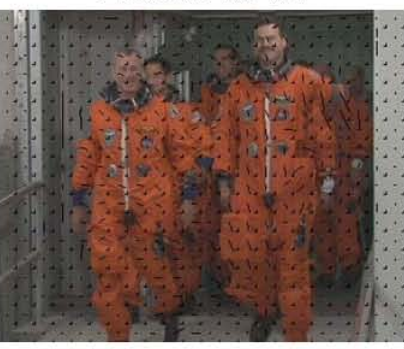

Frame No. 33

(a)

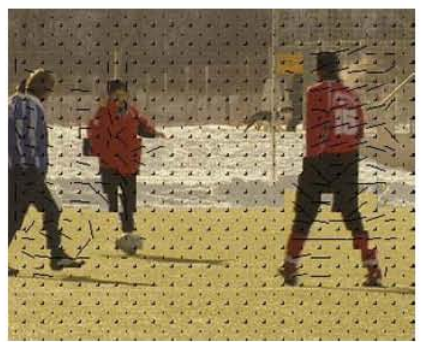

Frame No. 2

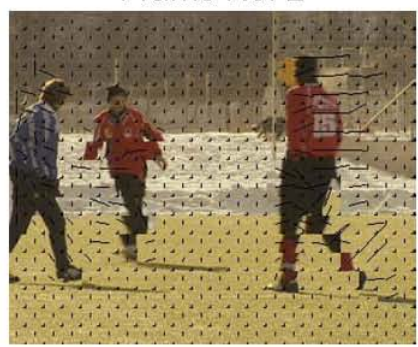

Frame No. 3

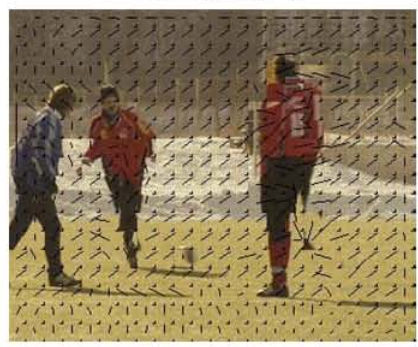

Frame No. 4

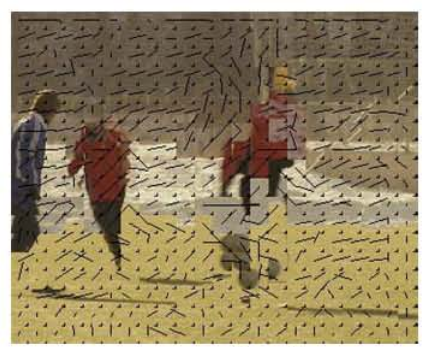

Frame No. 5

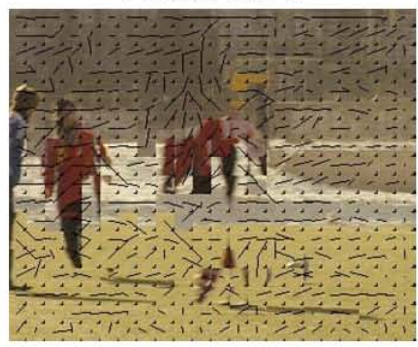

Frame No. 6

(b)
Figure 2: Examples of unreliable MV fields due to (a) abrupt illumination change (CIF Crew 15 fps) and (b) a low frame rate (CIF Soccer $7.5 \mathrm{fps}$ ). 


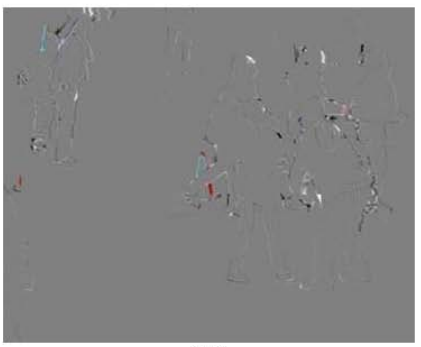

(a)

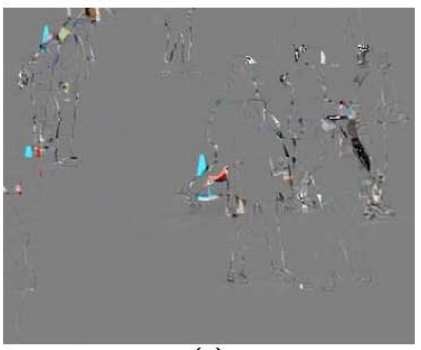

(c)

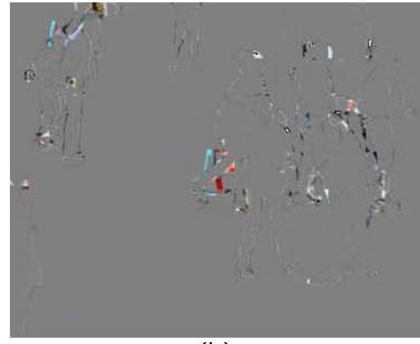

(b)

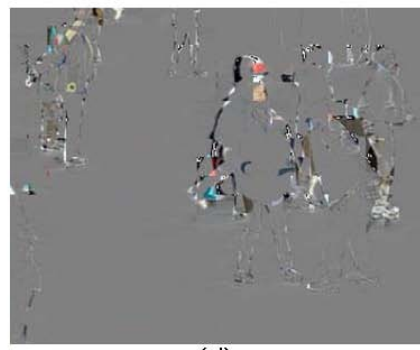

(d)

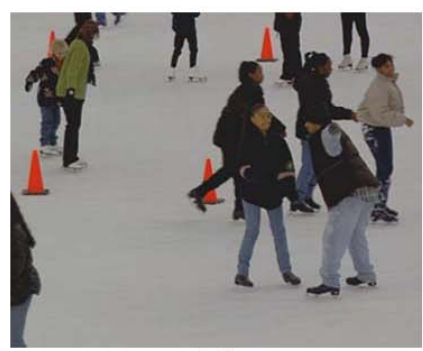

(e)

Figure 3: Motion residual of the Ice sequence: (a) $60 \mathrm{fps}$ (b) $30 \mathrm{fps}$ (c) 15 fps (d) 7.5 fps and (e) the original frame.

\section{Proposed Solution}

\section{A. Abrupt Illumination Change Detection and Local Intensity Adjustment}

An abrupt change in lighting condition introduces a significant shift in an average intensity level of the effected frame. Therefore, in order to detect this effect, frame distance based on the first- and the second-order differences of frame luminance histogram are computed.

$$
D^{n}(t)=\frac{1}{N} \sum_{i=0}^{B}\left|h_{i}^{n}(t)-h_{i}^{n}(t+1)\right|, n=1,2
$$

Where $D^{1}(t)$ and $D^{2}(t)$ denote the first- and the secondorder differences of the luminance histogram of frame $t$, respectively. $h_{i}^{1}(t)$ and $h_{i}^{2}(t)$ are the $\mathrm{i}^{\text {th }}$ cumulative histogram of frame $t$ and gradient frame $t$, respectively. $B$ is the number of bin for histogram and $\mathrm{N}$ is the total pixel number in each frame. The gradient frame is essentially a frame difference with threshold and thus, its frame distance is equivalent to the second-order differences of frame luminance histogram. The example of gradient frame compared to normal frame is presented in Fig. 4. The number of histogram bin can be adjusted lower to control the sensitivity of measured variable to noise such as camera and object movement. Then, after frame distance measurement, an adaptive thresholding technique [12] is used to determine the signal peak location. The adaptive threshold $T(t)$ is essentially a moving averaging window with an adjustable sensitivity parameter on data variance. It is described as follows

$$
\begin{gathered}
T(t)=\mu(t)+\alpha \cdot \sigma(t) \\
\mu(t)=\frac{1}{L} \sum_{i \in W} D(i) \\
\sigma^{2}(t)=\frac{1}{L} \sum_{i \in W}(D(i)-\mu(t))^{2}
\end{gathered}
$$

Where $\alpha$ is a scale factor, $\mu(t)$ and $\sigma^{2}(t)$ is the sample mean and sample variance of data $D$ within window $\mathrm{W}$ of length $L$ that has a center at frame $t$. A large luminance change is claimed when we detect simultaneous peak signals within the same frame. Afterwards, the local intensity difference ( $I_{\text {diff }}$ ) of each macroblock is measured by computing the average luminance difference of all macroblocks within a neighboring area to their corresponding location in the previous frame as:

$$
I_{\text {diff }}(m, n)=\frac{1}{M} \sum_{i=m-1}^{m+1} \sum_{j=n-1}^{n+1}\left|I_{a v}(i, j, t)-I_{a v}(i, j, t+1)\right|,
$$

where $I_{\text {diff }}(i, j, t)$ is the average intensity of macroblock $(\mathrm{i}, \mathrm{j})$ of frame $t$ and $M$ is the number of macroblocks in the neighbor of consideration. Then, the measured parameters are used to adjust the pixel value of detected frame to reduce the irregular intensity difference introduced by abrupt illumination change. Instead of using globally intensity adjustment, we employ locally intensity adjusting technique due to the fact that the considered lighting effect has an uneven influence on each area. For instance, intensity of foreground and object with reflective texture is highly affected by camera flash compared to object in background.
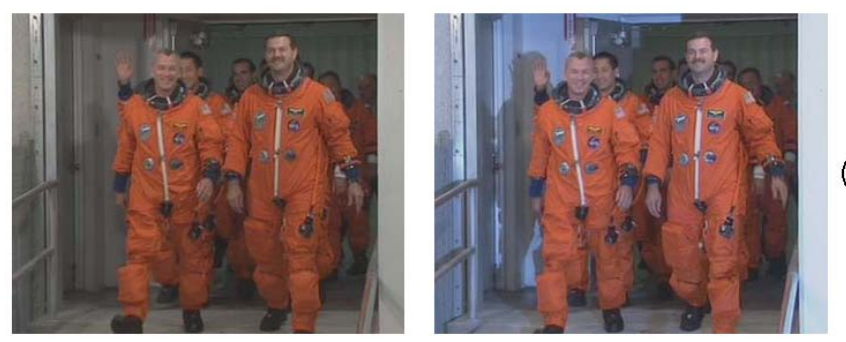

(a)
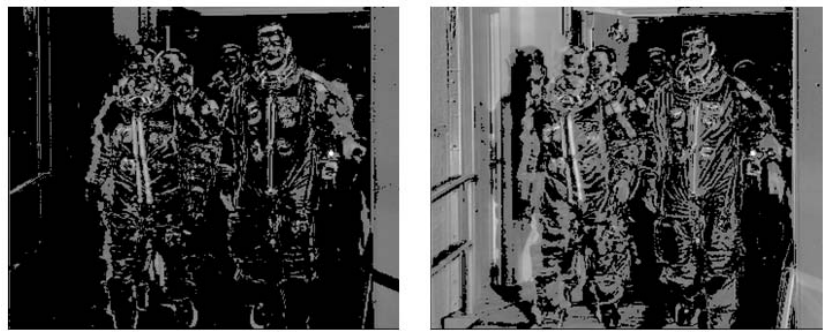

Frame no. 30

Figure 4: Example of gradient frame - frame no. 29\&30 (CIF crew 15 fps) (a) normal frame and (b) gradient frame. 


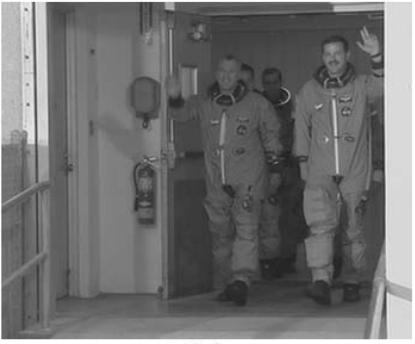

(a)

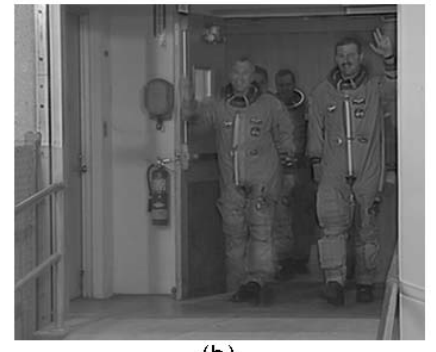

(b)
Figure 5: Example of local intensity adjustment - frame no. 3 (CIF crew 15 fps) (a) before processing and (b) after processing.
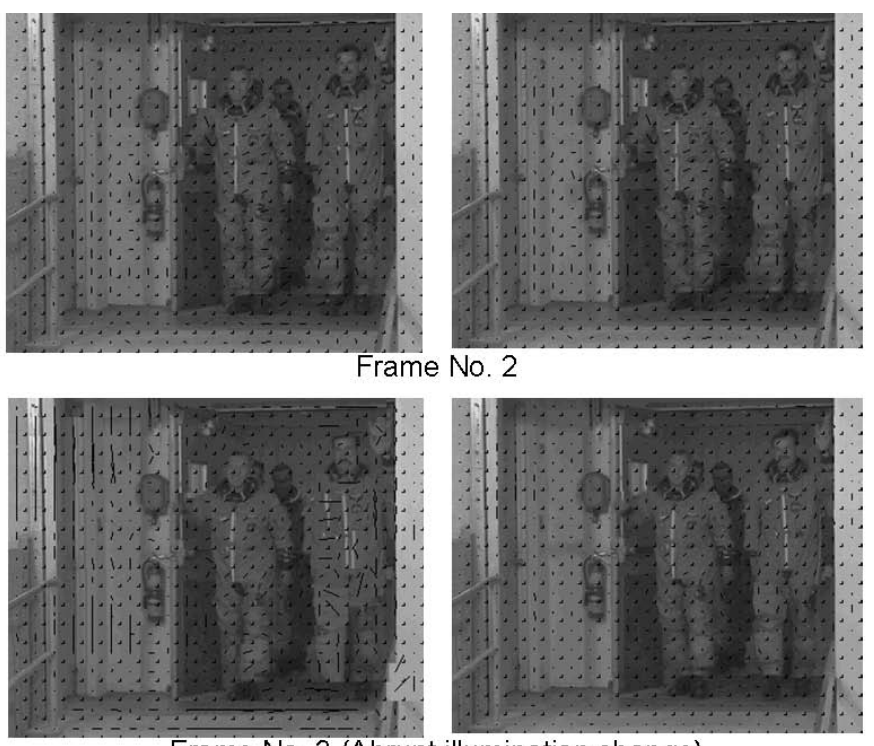

Frame No. 3 (Abrupt illumination change)

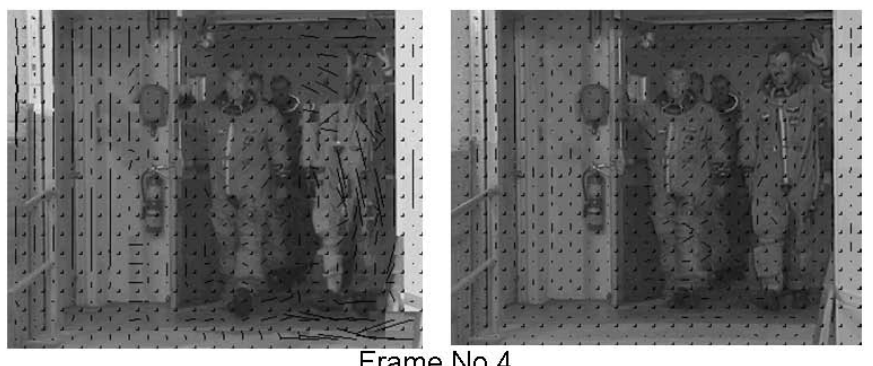

(a)

(b)

Figure 6: Example of motion vector field - frame no. 3-5 (CIF crew 15 fps) (a) before processing and (b) after processing.

The example result (Frame no.3 of crew sequence) of local intensity adjustment is illustrated in Fig. 5 and the comparison of corresponding MV fields (before and after the processing) is presented in Fig 6. In Fig. 5, the intensity adjusted frame experiences a blurring effect due to an averaging operation but its overall intensity level is much closer to that of the adjacent frames. In Fig. 6, after applying local intensity adjustment, the temporal continuity of $\mathrm{MV}$ field is restored and motion information becomes consistent with actual object motion.

\section{B. Proposed FRUC algorithm}

As discuss in previous section, due to a wider temporal gap between two adjacent frames of low frame rate video, most FRUC algorithms that only exploit temporal correlation and aim to maximize temporal continuity of up-sampled video will not be able to perform well. Therefore, we propose FRUC algorithm based on the first- and the second-order translational model that consider not only temporal continuity but also spatial smoothness of the interpolated frame.

For low frame rate video, the linear translational model assumption may not hold for all macroblock. However, there are still locations where this assumption may apply such as stationary background and slow-moving objects, etc. In order to identify them, we use the first and the second order forward and backward linear motion projection as described in Eq. (6)(9)

$$
\begin{aligned}
f_{F P, t+1}^{1}\left(P_{t}(m, n)\right) & =P_{t+1}\left(m+M V X_{t}(m, n), n+M V Y_{t}(m, n)\right) \\
f_{B P, t}^{1^{S t}}\left(P_{t+1}(m, n)\right) & =P_{t}\left(m+M V X_{t+1}(m, n), n+M V Y_{t+1}(m, n)\right) \\
f_{F P, t+1}^{2^{n d}}\left(P_{t}(m, n)\right) & =P_{t+1}\left(m+M V X_{t}(m, n)-M V X_{t}^{\prime}(m, n)\right. \\
& \left., n+M V Y_{t}(m, n)-M V Y_{t}^{\prime}(m, n)\right) \\
f_{B P, t}^{2^{n d}}\left(P_{t+1}(m, n)\right) & =P_{t}\left(m+M V X_{t+1}(m, n)-M V X_{t+1}^{\prime}(m, n)\right. \\
, n+M V Y_{t+1} & \left.(m, n)-M V Y_{t+1}^{\prime}(m, n)\right)
\end{aligned}
$$

where $f_{F P, t}^{N^{t h}}$ and $f_{B P, t}^{N^{t h}}$ are the $N^{t h} \operatorname{order}(N=1,2)$ forward and backward macroblock projection onto frame $t$, respectively, $P_{t}(m, n)$ is macroblock at location $(\mathrm{m}, \mathrm{n})$ in frame $\mathrm{t}$ and its corresponding horizontal and vertical MV are denoted by $M V X_{t}(m, n)$ and $\operatorname{MVY}_{t}(m, n)$, respectively. $M V X_{t}^{\prime}(m, n)$ and $M V Y_{t}^{\prime}(m, n)$ denote an acceleration of horizontal and vertical motion and obtained by tracking the trajectory path as shown in Fig. 7. Motion estimation will use previous and next frame as a reference frame for forward and backward projection, respectively. After the projection, we compare the actual macroblock with the projected image. If their first order difference is within the detection threshold, this macroblock is identified as a linear translation macroblock, and the projected images on the interpolated frame can be used for processing. If there exists an overlapping region of several projected images, pixel averaging is employed.

In the next stage, we use the second-order translational model to detect locations with non-linear motion. The secondorder detection threshold is set lower than the first-order threshold to ensure the accuracy of interpolated areas. Unlike many existing motion projection-based FRUC algorithms such as [3], the proposed technique does not force every macroblock to select the best linear motion trajectory and/or interpolate the MV of every location since these strategies usually result in low quality of interpolated frames when the linear translation assumption fails. For practical application, we can also reduce the requirement on computational complexity and frame buffer storage by using only the firstand the second-order forward translational model. In the final 
step, the proposed FRUC is to use technique similar to overlapped boundary block matching (OBMA) [13] - [15] to fill in an area which is left by the second stage. The MV candidate is obtained from the neighboring area of the corresponding macroblocks in frames $t$ and $t+1$. This criterion is introduced to ensure the spatial continuity of interpolated frames. An example of interpolated frame obtained after each step of proposed FRUC algorithm is shown in Fig. 8 (CIF ice $7.5 \mathrm{fps}$ ). As shown in this figure, most of background area and very slow moving object have been interpolated after the first two steps (Fig. 8(a) and 8(b)). Most of the remaining uninterpolated areas consist of fast moving object, object boundary and occlusion area. The translational models used in our proposed FRUC algorithm are limited and cannot always capture the motion of fast moving object, especially under an influence of camera motion. For object boundary and occlusion area, it is unlikely that the projected macroblock will be able to find the closed match within the projected area. Nevertheless, as we discuss earlier, our proposed algorithm does not aim to fit every area with translation model. These remaining areas are interpolated using OBMA and the result is shown in Fig. 8(c). As discuss in [15], this technique can preserve the continuity of object boundary. In addition, it is also able to interpolate uncovered and to-be-cover occlusion area since OBMA search the matching candidates from all reference frame in both forward and backward temporal direction. Another example of interpolated frame from proposed FRUC algorithm is shown in Fig. 9 (CIF city 7.5fps). According to Fig. 9(a), the majority area of interpolated frame is identified by the first stage of proposed algorithm since this video sequence complies with linear translation model due to the fact that its content consists mainly of stationary solid object (building). Additionally, because of the existing camera motion, the first-order translation model is unable to interpolate the entire frame and as we can observe from Fig. 9(b), the second-order translation model doesn't contribute much information for the interpolated frame. Nevertheless, OBMA can effectively incorporate spatial information (edge and texture) to interpolate the missing data as shown in Fig. 9(c).

\section{OTHER LOW COMPLEXITY FRUC AlgORITHMS}

In this section, we present the concise detail of six other low complexity FRUC algorithms that will be used as performance benchmark, i.e. Frame Repetition (FR), Frame Averaging (FA), Motion copy (MC) and three motion projection based techniques - Forward (FP), Backward (BP) and Bi-direction (BIP). For FR, the current frame is used directly as an interpolated frame. This technique has the lowest complexity and can be considered as a lower bound of performance measurement since it doesn't actually increase the perceived frame rate of the processed video. FA acquires the interpolated frame by weight averaging two adjacent frames. The weighting parameter can be adaptively adjusted based on frame content but in this work, simple averaging operation will be employed. MC interpolated each macroblock

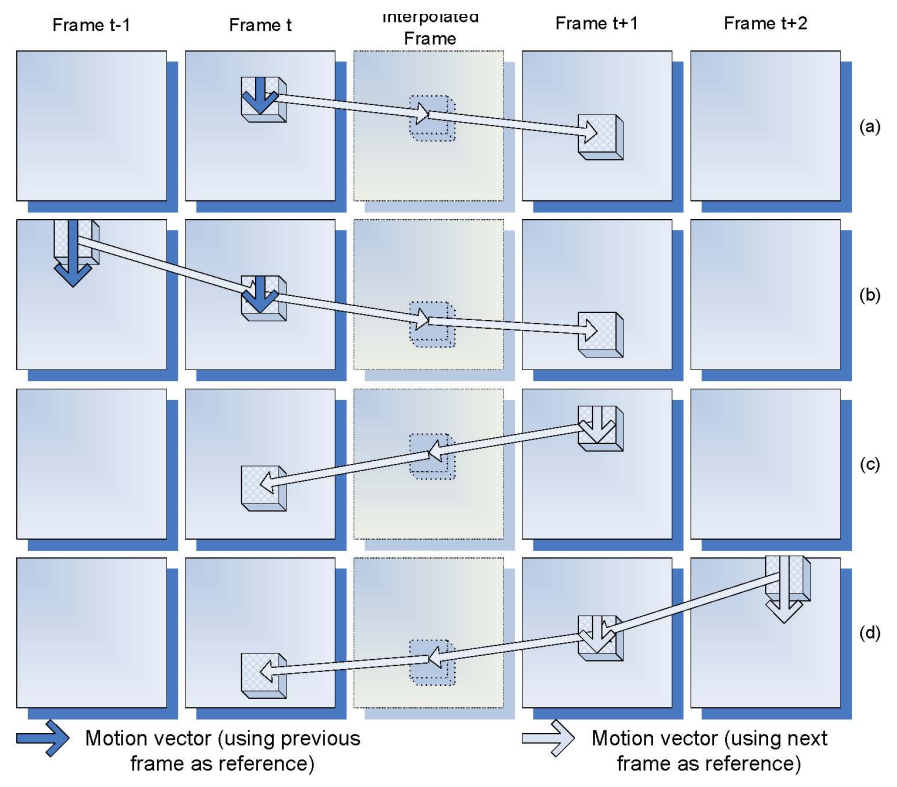

Figure 7: Example of motion projected macroblock (a) the first-order forward translation macroblock (b) the second-order forward translational macroblock (c) the first-order backward translation macroblock and (d) the second-order backward translational macroblock.

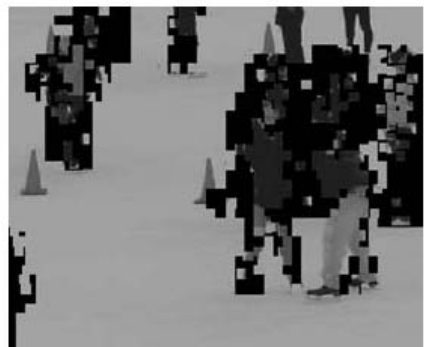

(a)

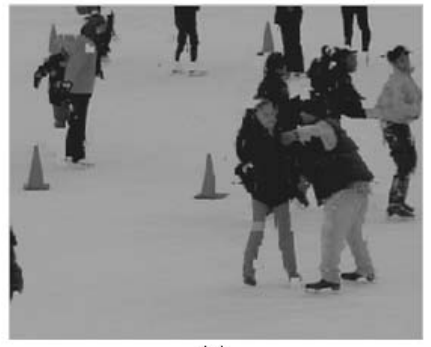

(c)

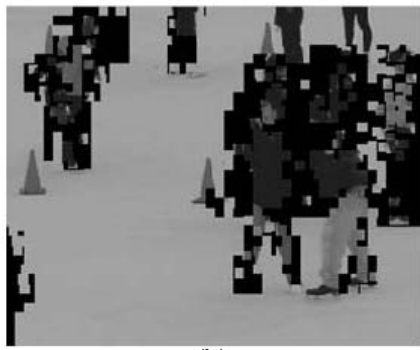

(b)

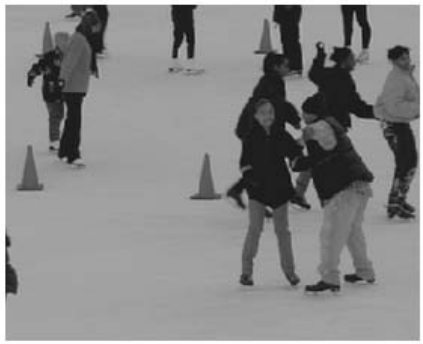

(d)
Figure 8: Illustration of proposed FRUC interpolation process (CIF ice 7.5fps) (a) after the first-order translation detection (b) after the secondorder translation detection (c) after OBMA and (d) the actual frame

in the up-sampled frame based on a scaled MV obtained from the corresponding macroblock in the current frame. In this work, the scaled MV has quarter-pel resolution. The motion projection based technique relies on motion information of two adjacent frames to project each macroblock of these frames onto the interpolated frame. Normally, there exists an overlapped area of several projected macroblock as well as a blank pixel that doesn't occupy by any projected data. For the overlapped area, simple averaging is usually applied to merge the overlapped information while simple motion interpolation 


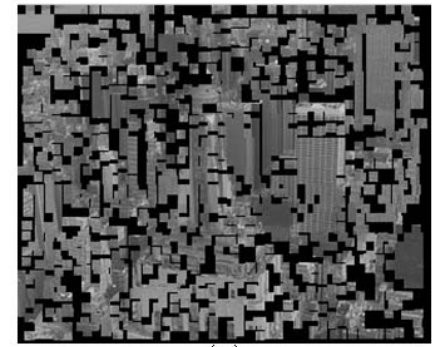

(a)

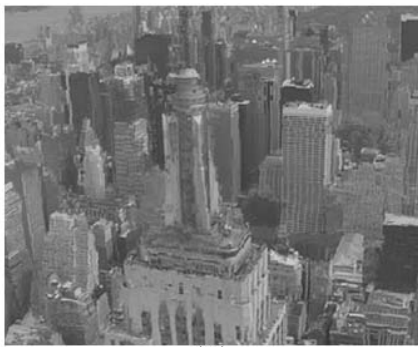

(c)

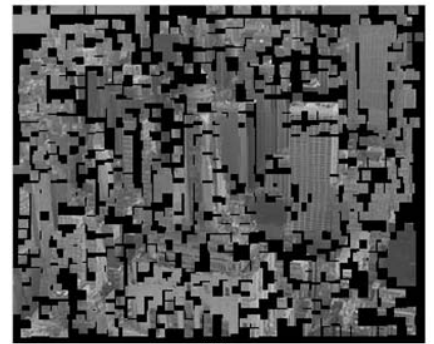

(b)

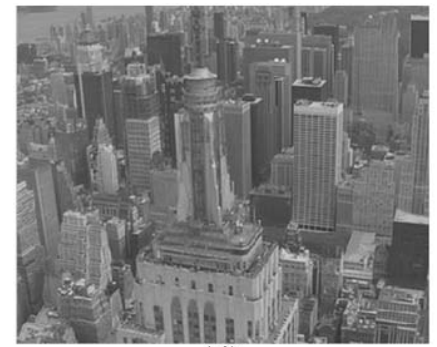

(d)
Figure 9: Illustration of proposed FRUC interpolation process (CIF city 7.5fps) (a) after the first-order translation detection (b) after the secondorder translation detection (c) after OBMA and (d) the actual frame

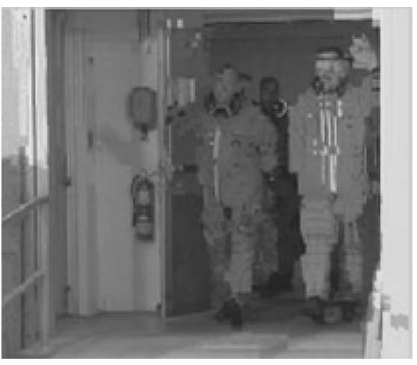

(a)

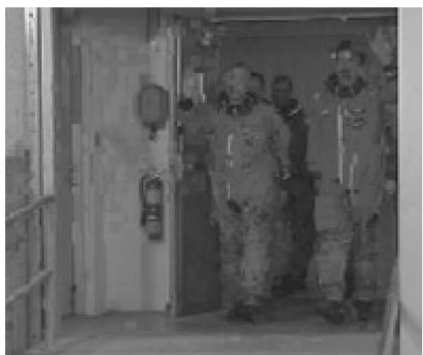

(c)

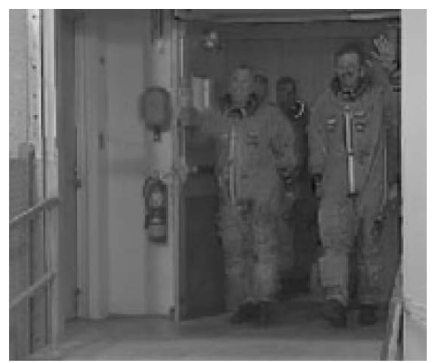

(b)

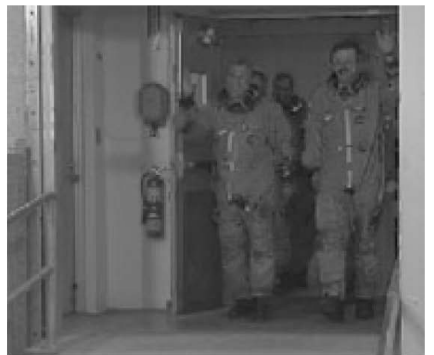

(d)
Figure 10: Interpolated frames in the presence of flashlight: (a) MI preprocessing (19.96dB) (b) MI post-processing (26.48dB) (c) Proposed FRUC pre-processing (26.12dB) and (d) Proposed FRUC post-processing (28.68dB)

technique and averaging mask can be used to fill out the missing data. In general, motion projection based technique can be performed in both pixel and macroblock level.In [16], it is shown that pixel-level algorithm yields a better performance but also has a higher computational complexity. In favor of low complexity, macroblock-level algorithm will be used in our experiment and in order to obtain a better spatial correlation, OBMA will be utilized to fill out the missing data. FP and BP derives its name from the projection direction while BIP obtains the interpolated frame by weight

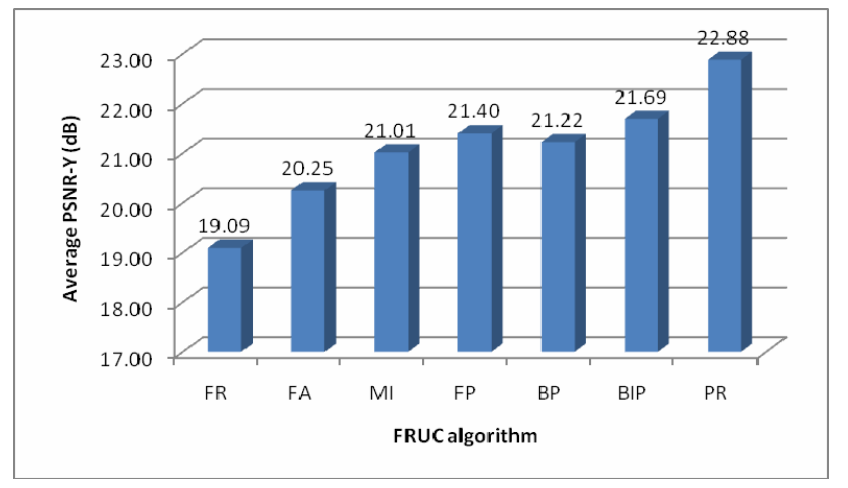

Figure 11: Performance comparison of several low-complexity FRUC algorithms

averaging the interpolation results from FP and BP. Similar to FA, various merging scheme can be applied [17] but simple weighting will be used.

\section{V.EXPERIMENTAL RESULTS}

All low complexity FRUC algorithms presented in previous section can be applied to any up-sampling factor but for sake of simplicity, we consider 1-to-2 frame rate up-sampling throughout our experiments. To get the ground truth, we down-sample test sequences of $15 \mathrm{fps}$ to sequences of a low frame rate (i.e., $7.5 \mathrm{fps}$ ). Five different video sequences (CIF) are used to test the proposed algorithm. They are city, crew, ice, soccer and football.

\section{A. Abrupt Illumination Change}

The effect of a local intensity adjusting technique on MI and proposed FRUC algorithm (PR) can be observed from Fig. 10. According to experimental result, $\mathrm{MI}$ and $\mathrm{PR}$ gain an average PSNR improvement of 3.22 and $1.45 \mathrm{~dB}$, respectively. MI obtains a larger performance increase over PR due to the fact that its interpolation process relies solely on temporal information while PR considers both temporal and spatial condition. In addition, the perceptual quality of interpolated frame is greatly improved after the proposed processing is employed. The blocky artifact that exists in Fig. 10(a) and 10 (c) is a result of inaccurate MV caused by camera flash.

\section{B. Low frame rate}

The performance comparison of several low-complexity FRUC algorithms is demonstrated in Fig. 11. The presented PSNR is acquired from an average results of five testing video sequence. Based on obtained results, FA is approximately $1.16 \mathrm{~dB}$ better than the performance's lower bound (FR) but it has a very poor perceptual quality. PR gains an average PSNR improvement of $3.79 \mathrm{~dB}, \quad 2.63 \mathrm{~dB}$ and $1.87 \mathrm{~dB}$ over FR, FA and MI interpolation scheme, respectively. All three motion projection based techniques (FP, BP and BIP) yield a similar level of performance and is approximately $1.25 \mathrm{~dB}$ lower than PR. In order to evaluate the perceptual quality of interpolated frame, three examples from city, ice and soccer sequence are presented in Fig. 12, 13 and 14, respectively. Each of these 


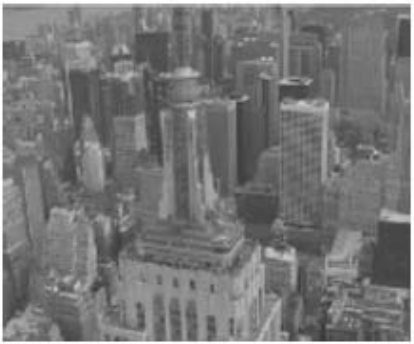

(a)

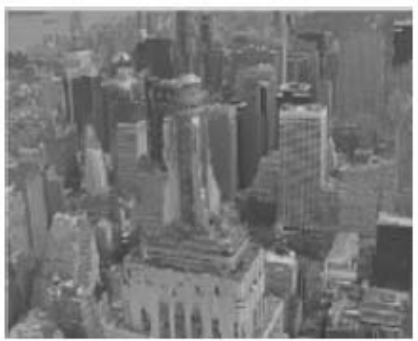

(c)

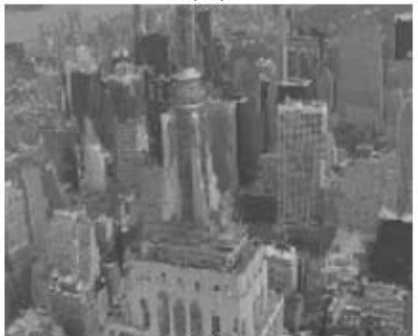

(e)

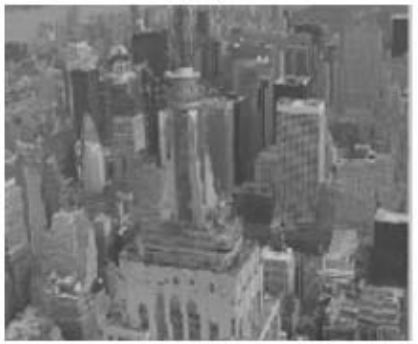

(g)

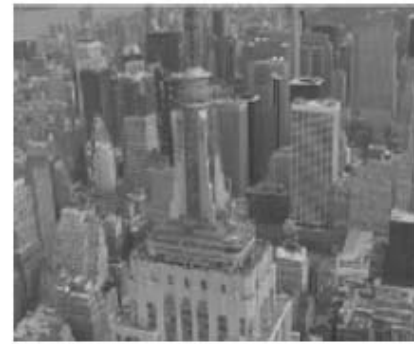

(b)

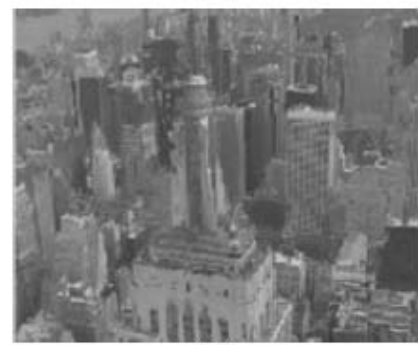

(d)

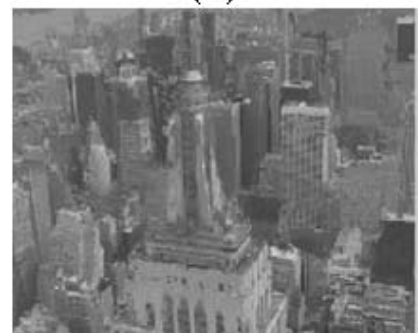

(f)

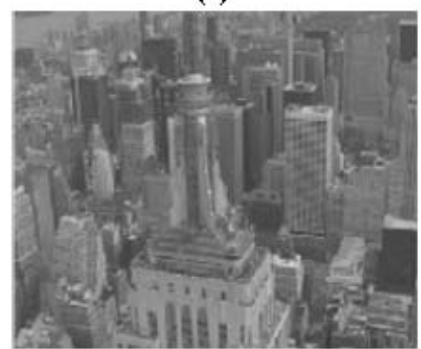

(h)

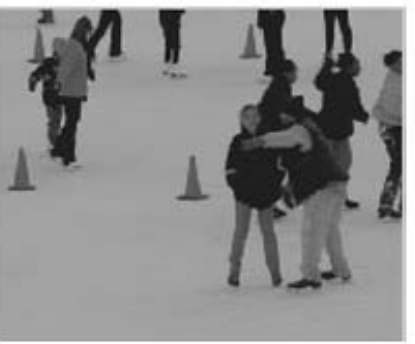

(a)

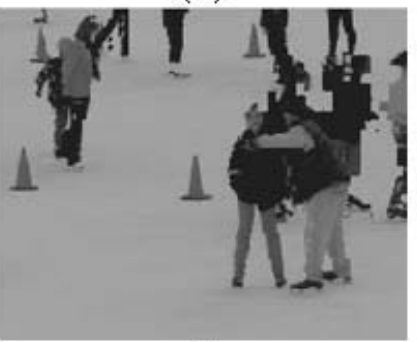

(c)

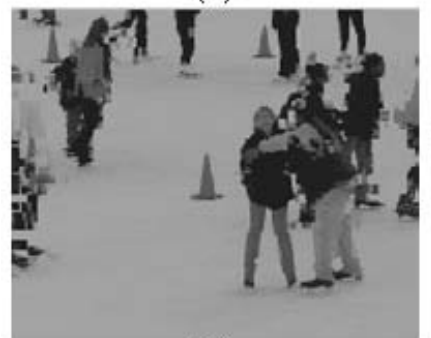

(e)

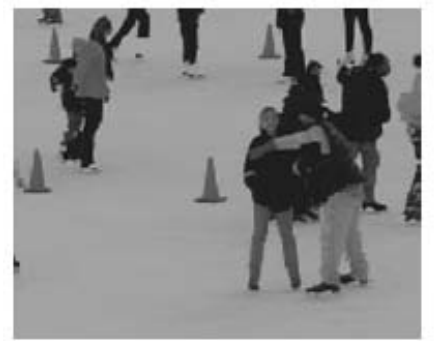

(g)

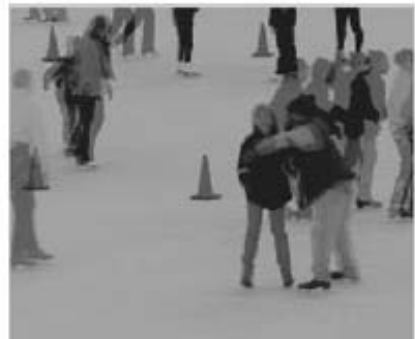

(b)

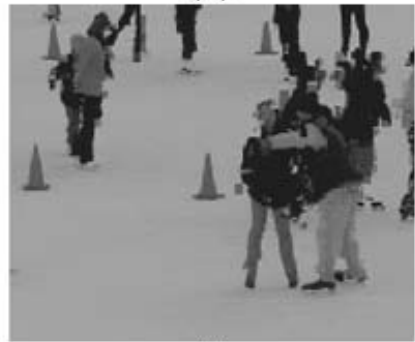

(d)

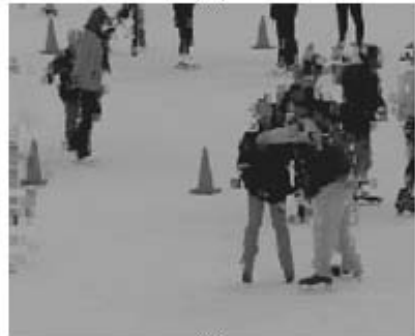

(f)

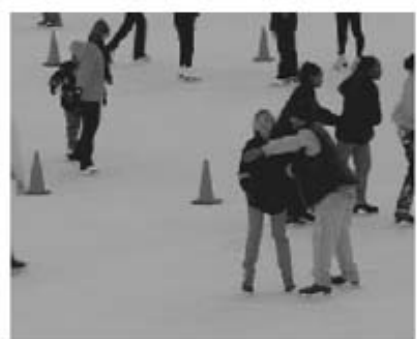

(h)
Figure 12: Example of interpolated frames (CIF city7.5fps) (a) FR (b) FA (c) MI (d) FP (e) BP (f) BIP (g) Proposed FRUC and (h) actual frame

video sequences has unique character which significantly influences the performance of FRUC algorithms. For city sequence, it consists mainly of stationary solid objects and has slight camera motion. With these properties, most FRUC algorithm can perform very well even under a very low frame rate condition as we can observe from Fig. 12. On the other hand, ice sequence experiences no camera motion but consists of several fast moving objects. All three motion projection based techniques severely suffer from an inaccurate macroblock projection (Fig. 13(d), 13(e) and 13(f)) since they indiscriminately apply projection model to all macroblock. On the contrary, interpolated frame from PR has a much better perceptual quality due to the spatial smoothness criterion exerted by OBMA. Among all these examples, soccer sequence posts the greatest challenge since it is affected by fast camera panning and fast object motion. The average
Figure 13: Example of interpolated frames (CIF ice 7.5fps) (a) FR (b) FA (c) MI (d) FP (e) BP (f) BIP (g) Proposed FRUC and (h) actual frame

PSNR of interpolated frame from soccer sequence is about 2.85 and $1.32 \mathrm{~dB}$ lower than that of city and ice sequence, respectively. According to example results shown in Fig. 14, PR produces the best perceptual quality but there still exist some erroneous details such as the number of soccer player. Nevertheless, these errors are difficult to be perceived when video sequence is displayed at $15 \mathrm{fps}$.

\section{Conclusion}

In this work, we identify and analyze two challenging problems for FRUC. For abrupt illumination change, we propose a low complexity processing techniques to ensure robust MV estimation and, as a result, the quality of the upconverted video sequence under this condition has improved greatly. To handle the second challenge, the proposed FRUC algorithm, which utilizes the first and the second translational 


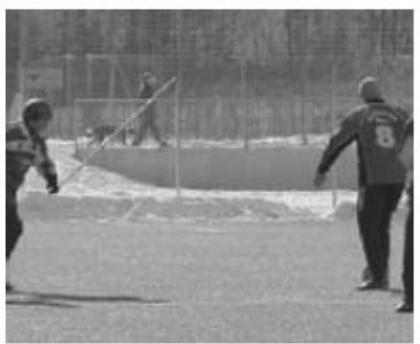

(a)

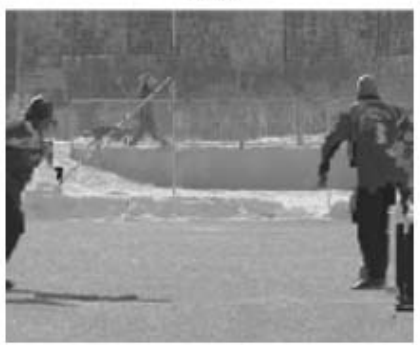

(c)

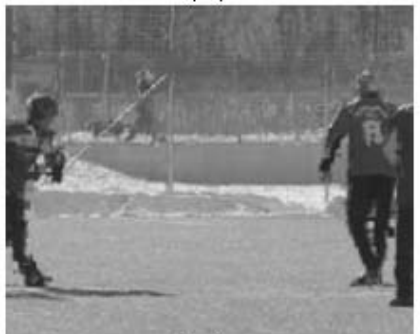

(e)

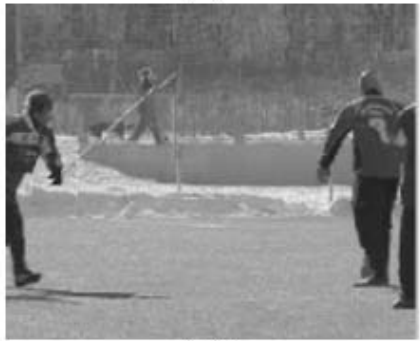

(g)

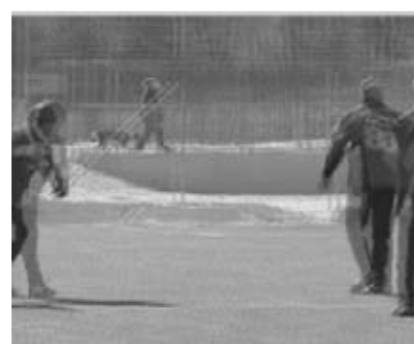

(b)

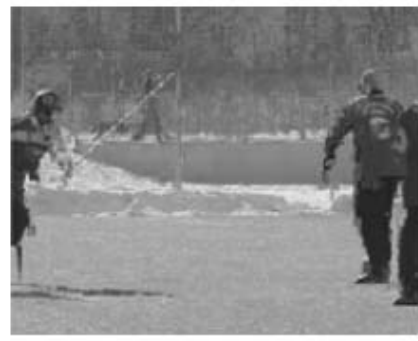

(d)

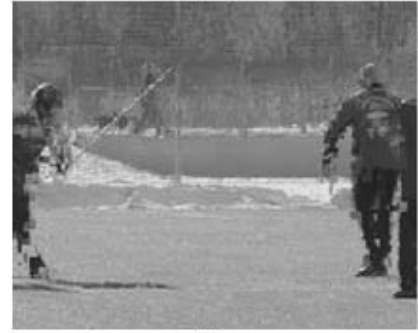

(f)

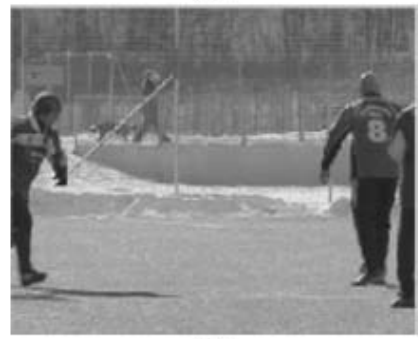

(h)
Figure 14: Example of interpolated frames (Soccer ice 7.5fps) (a) FR (b) FA (c) MI (d) FP (e) BP (f) BIP (g) Proposed FRUC and (h) actual frame

model as well as spatial smoothness criterion, is presented. Based on the experimental results, it is proven to perform much better than other low-complexity FRUC benchmarks under low frame rate constraint.

\section{REFERENCES}

[1] T. Thaipanich, P.-H. Wu and C.-C. J. Kuo, "Robust Video Frame Rate Up-Conversion (FRUC) Techniques," to be appear in International Conference on Consumer Electronics (ICCE2009), January 13th, 2008

[2] B.W. Jeon, G.I. Lee, S.H. Lee and R.H. Park, "Coarse-To-fine Frame Interpolation For Frame Rate Up-Conversion Using Pyramid Structure," IEEE Transaction on Consumer Electron, Vol. 49, Issue 3, pp. 499-508.

[3] B.-T. Choi, S.-H. Lee, and S.-J. Ko, "New Frame Rate Up-Conversion Using Bi-directional Motion Estimation," IEEE Transactions on Consumer Electronics, Vol. 46, Issue 3, August 2000, pp. 603-609
[4] S. Han and J. Woods, "Frame-Rate Up-Conversion Using Transmitted Motion And Segmentation Fields For Very Low Bit-Rate Coding," Proceedings of IEEE International Conference on Image Processing (ICIP), 1997, Santa Barbara, CA, Oct. 1997.

[5] K. Kawaguchi and S. K. Mitra, "Frame Rate Up-Conversion Considering Multiple Motion," Proceedings of IEEE International Conference on Image Processing (ICIP), Santa Barbara, CA, Oct.1997

[6] R. L. Lagendijk and M. I. Sezan, "Motion Compensated Frame Rate Conversion Of Motion Pictures," Proceedings of IEEE International Conference on Acoustics, Speech and Signal Processing (ICASSP), Vol. 3, pp. 453-456, 1992.

[7] S.-H Lee, O.-J. Kwon and R.-H. Park, "Weighted-Adaptive MotionCompensated Frame Rate Up-Conversion," IEEE Transactions on Consumer Electronics, Vol. 49, Aug. 2003, pp. 485-492

[8] K. Hilman, H. W. Park and Y. M. Kim, "Using Motion-Compensated Frame-Rate Conversion For The Correction Of 3:2 Pulldown Artifacts In Video Sequences", IEEE Transactions on Circuits and Systems for Video Technology, Vol.10, (3), Sept. 2000, pp. 869-877

[9] R. Castagno, P. Haavisto, and G. Ramponi, "A Method For Motion Adaptive Frame Rate Up-Conversion," IEEE Transactions on Circuits and Systems for Video Technology, Vol. 6, Oct. 1996, pp. 436 - 446

[10] G. Dane and T.Q. Nguyen, "Motion Vector Processing For Frame Rate Up Conversion," Proceedings of IEEE International Conference on Acoustics, Speech and Signal Processing (ICASSP), Vol. 3, pp. 309312, May 2004

[11] S. Fujiwara and A. Taguchi, "Motion-Compensated Frame Rate UpConversion Based On Block Matching Algorithm With Multi-Size Blocks," Proceedings of International Symposium on Intelligent Signal Processing and Communication Systems (ISPACS), Page(s): 353 - 356, Dec. 2005.

[12] H.J. Zhang, A. Kankanhalli and S.W. Smoliar, "Automatic Partitioning Of Full-motion Video," Multimedia Systems (1993) Vol. 1, No. 1, pp. $10-28$.

[13] T. Thaipanich, P.-H. Wu, C.-C. Jay Kuo, "Video Error Concealment With Outer And Inner Boundary Matching Algorithms," Conference on Applications of Digital Image Processing, SPIE Optics and Photonics, San Diego, CA, USA, Aug. 26-30, 2007.

[14] T. Thaipanich, P.-H. Wu, C.-C. Jay Kuo, "Low-Complexity Mobile Video Error Concealment Using OBMA," International Conference on Consumer Electronics (ICCE), Las, Vegas, NV, USA, Jan. 2008.

[15] T. Thaipanich, P.-H. Wu and C.-C. J. Kuo, "Low-Complexity Video Error Concealment For Mobile Applications Using OBMA," IEEE Transactions on Consumer Electronics, Vol. 54, Issue 2, May 2008, pp. 753-761.

[16] Y. Chen, K. Yu, J. Li, and S. Li, "An Error Concealment Algorithm For Entire Frame Loss In Video Transmission," Presented at the Picture Coding Symposium, San Francisco, CA, Dec 2004.

[17] S. Belfiore, M. Grangetto, E. Magli, and G. Olmo, "An Error Concealment Algorithm For Streaming Video," Proceedings of IEEE International Conference on Image Processing (ICIP), 2003, pp. 649652.

[18] S. Belfiore, L. Crisi, M. Grangetto, E. Magli, and G. Olmo, "Robust And Edge-Preserving Video Error Concealment By Coarse-To-Fine Block Replenishment," Proceedings of IEEE International Conference on Acoustics, Speech and Signal Processing (ICASSP), 2002.

[19] P. Baccichet and A. Chimienti, "A Low Complexity Concealment Algorithm For The Whole-Frame Loss In H.264/AVC," Presented at the MMSP, 2004.

[20] Q. Peng, T. W. Yang, and C. Q. Zhu, "Block-Based Temporal Error Concealment For Video Packet Using Motion Vector Extrapolation," Proceedings of IEEE International Conference on Communications, Circuits And System (ICASSP), West Sino Expo., 2002, pp. 10-14.

[21] H. Schwarz, D. Marpe and T. Wiegand, "Overview Of The Scalable Video Coding Extension Of The H.264/AVC Standard, IEEE Transactions on Circuits and Systems for Video Technology, Vol. 17, No. 9, pp. 1103-1120, Sept. 2007

[22] T. Wiegand, G. J. Sullivan, G. Bjøntegaard, and A. Luthra, "Overview Of The H.264/AVC Video Coding Standard," IEEE Transactions on Circuits and Systems for Video Technology, Vol. 13, No. 7, pp. 560576, Jul. 2003.

[23] K. Simonyan, S. Grishin and D. Vatolin , "VirtualDub MSU Motion Estimation Filter - version 1.0" 


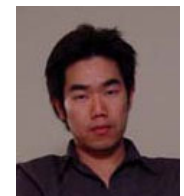

Tanaphol Thaipanich received his B.Eng degree in Electrical Engineering at Chulalongkorn University in 2002 and M.S. degree in Electrical Engineering at University of Southern California in 2003. He is currently a Ph.D. student in Department of Electrical Engineering at University of Southern California. He is a member of Prof. Kuo research group. His research interests include computer vision and error detection and concealment in video data.

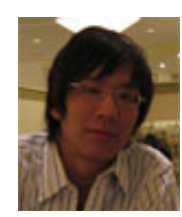

Ping-Hao Wu is a Ph.D. student in the Electrical Engineering Department, University of Southern California. He received his B.S. degree in Electrical Engineering and the M.S. degree in Communication Engineering from National Taiwan University in 2004 and 2006, respectively. He is currently a member of Prof. Kuo's research group. His research interests include digital signal processing and image/video coding.

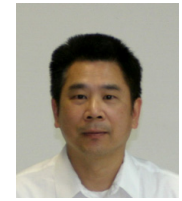

C.-C. Jay Kuo received the B.S. degree from the National Taiwan University, Taipei, in 1980 and the M.S. and Ph.D. degrees from the Massachusetts Institute of Technology, Cambridge, in 1985 and 1987, respectively, all in Electrical Engineering. $\mathrm{He}$ is Director of the Signal and Image Processing Institute (SIPI) and Professor of Electrical Engineering, Computer Science and Mathematics at the University of Southern California (USC). His research interests are in the areas of digital image/video analysis and modeling, multimedia data compression, communication and networking and multimedia database management. Dr. Kuo has guided about 87 students to their Ph.D. degrees and supervised 20 postdoctoral research fellows. He is a co-author of about 140 journal papers, 740 conference papers and 9 books. Dr. Kuo is a Fellow of IEEE and SPIE. He is Editor-in-Chief for the Journal of Visual Communication and Image Representation, and Editor for the Journal of Information Science and Engineering, LNCS Transactions on Data Hiding and Multimedia Security and the EURASIP Journal of Applied Signal Processing. Dr. Kuo received the National Science Foundation Young Investigator Award (NYI) and Presidential Faculty Fellow (PFF) Award in 1992 and 1993, respectively. 